\title{
The Role of Attention in Increasingly Autonomous Driving
}

\section{Trent Victor}

\section{Distinguished Keynote Luncheon Speaker}

Trent is Senior Technical Leader Crash Avoidance at Volvo Cars Safety Centre, and Adjunct Professor at Chalmers University of Technology in Gothenburg, Sweden. At Volvo Cars his role is to identify and define future leading edge safety needs within crash avoidance. His role provides leadership in safety analysis and research, supporting development of safety requirements and strategies and to drive human related aspects of safety design in a broader perspective, using the possibilities with new active safety functions and systems. For example he is responsible for safety in Volvo's self-driving car program Drive Me. At Chalmers, he is very active in the analysis of naturalistic driving data at SAFER Vehicle and Traffic Safety Centre at Chalmers, where he recently was PI for a SHRP2 S08 naturalistic driving risk analysis of driver inattention. 